\title{
Efficacy, Adherence and Side Effects of PrEP for HIV-1 Prevention
}

\author{
Wanyi Fang ${ }^{1,2}$, Bongani I. Mphoyi ${ }^{1,2}$, Dineo R. Motake ${ }^{1,2}$, Jianxin Liu ${ }^{1,2}$, Wenshuang $\mathrm{Li}^{1,2}$, Fanghua Mei ${ }^{3}$, \\ Shenghan Lai ${ }^{1,4}$ \& Jun Wang ${ }^{1,2}$ \\ ${ }^{1}$ Department of Biomedicine and Biopharmaceuticals, Hubei University of Technology, Wuhan, Hubei Province, \\ China \\ ${ }^{2}$ National 111 Centre for Cellular Regulation and Molecular Pharmaceutics, Wuhan, Hubei, China \\ ${ }^{3}$ Hubei Provincial Academy of Preventive Medicine, Wuhan, Hubei, China \\ ${ }^{4}$ Department of Pathology, Johns Hopkins School of Medicine, Baltimore, Maryland, USA \\ Correspondence: Jun Wang, Department of Biomedicine and Biopharmaceuticals, Hubei University of \\ Technology, National 111 Centre for Cellular Regulation and Molecular Pharmaceutics, Wuhan, Hubei Province, \\ China. Tel: 86-1592-6414-738. E-mail: jun_wang@hbut.edu.cn
}

\begin{abstract}
Each year, approximately two million new HIV infections are reported worldwide. About one decade ago, a company called Gilead Sciences Inc. discovered a new HIV prevention method named as pre-exposure prophylaxis (PrEP). When high adherence was kept among high-risk population, PrEP efficacy could reach as high as $99 \%$. However, the adverse effects have been reported from time to time, including low efficacy in certain cases, adherence difficulties and medicinal side effects. In this review, we would summarize the progress of PrEP since its introduction in order to provide insights for HIV prevention.
\end{abstract}

Keywords: HIV, PrEP, FTC/TDF, MSM, Adherence, Side Effects

\section{Introduction}

Each year, approximately two million new HIV infections are reported worldwide (Anderson et al., 2012). New effective interventions are urgently needed (Krakower \& Mayer, 2015). A little over a decade ago, a company called Gilead Sciences Inc. developed a new HIV prevention method named as pre-exposure prophylaxis (PrEP). A pill called Truvada, which is the mixture of two components emtricitabine and tenofovir disoproxil fumarate (FTC/TDF) (Calabrese, Krakower, \& Mayer, 2017), has been introduced to the world since then. To date, Truvada or FTC/TDF is the most widely accepted drug for HIV prevention. FTC/TDF functions by preventing HIV-1 from replication after entering a human body. The mechanism is through inhibition of the activity of HIV-1 reverse transcriptase, which results in chain termination.

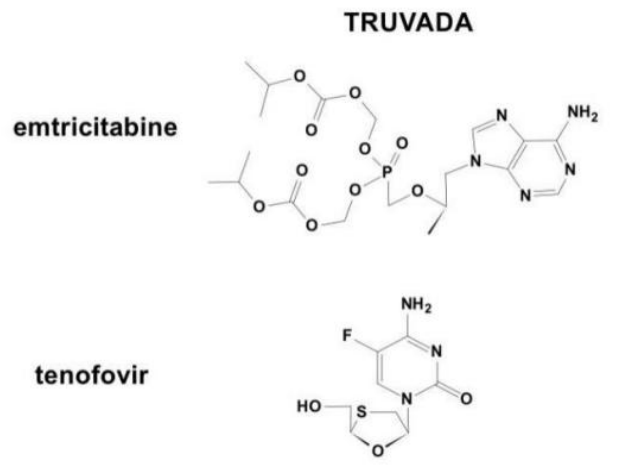

Figure 1. Chemical structure of emtricitabine and tenofovir disoproxil fumarate in Truvada

Since its approval by the United States Food and Drug Administration (FDA) in 2012 (Siegler et al., 2018), PrEP has been warmly embraced by many other countries. At the same time, a number of clinical trials have provided 
data to support this method, with efficacy in the range of 44-99\% (Anderson et al., 2012; Grant et al., 2010; Murnane et al., 2013). However, there are still challenges associated with PrEP due to numerous factors including lack of awareness of PrEP, high cost, poor adherence, risky sexual behavior and risk compensation, etc. (Hosek et al., 2013). To overcome these issues, a number of new strategies have been explored and implemented, including long-acting nanofluidic implants, modern monitoring strategies and improved screening algorithms (Sharma et al., 2019). In addition, side effects of PrEP have been recognized from time to time. In this paper, we summarized the status of PrEP efficacy, adherence and side effects between 2012 to 2019, according to a thorough search of literature with PubMed, Web of Science and Google scholar.

\section{Efficacy of PrEP}

According to results from previous trials and studies, PrEP has shown a wide range of efficacy performance. In studies of heterosexual men and women, men that have sex with men (MSM), injection-drug users, and couples in serodiscordant heterosexual relationships, efficacy was reported to be between $44 \%$ and $99 \%$ (Anderson et al., 2012; Grant et al., 2010; Murnane et al., 2013).

Table 1. Summary of PrEP studies between 2007 and 2018

\begin{tabular}{|c|c|c|c|c|}
\hline Study & Population & Dose & Risk reduction & References \\
\hline $\begin{array}{l}\text { Bangkok tenofovir study } \\
\text { group }\end{array}$ & Injection drug users & Daily & $\begin{array}{l}48.9 \% \text { ( } 74 \% \text { with ideal } \\
\text { adherence) }\end{array}$ & (Mimiaga et al., 2018) \\
\hline TDF2 & Heterosexual men and women & Daily & $62.20 \%$ & (Baeten et al., 2012) \\
\hline iPrEx study & MSM & Daily & $44 \%$ ( $92 \%$ with ideal adherence $)$ & (Grant et al., 2010) \\
\hline Partners PrEP Study & Serodiscordant heterosexual couples & Daily & $75 \%$ (90\% with ideal adherence) & (Molina et al., 2015) \\
\hline ANRS IPERGAY & MSM & ON-demand & $86 \%$ & (Janes et al., 2018) \\
\hline PROUD & Gay and other MSM & Daily & $86 \%$ & (Pyra et al., 2018) \\
\hline Observational cohort study & MSM & 18 pills per month & $97 \%$ & (Molina et al., 2017) \\
\hline STRAND Study & MSM & Daily & $99 \%$ & (Anderson et al., 2012) \\
\hline
\end{tabular}

In the early iPrEx study that began in 2007, the efficacy of PrEP was found to be relatively low, with $44 \%$ among MSM (Sanchez, Vivancos, \& Moreno, 2017). The STRAND study, which began a few years after iPrEx, analysed TFV-DP concentrations with respect to results from the iPrEx trial and concluded that risk reduction ratio increased with consistent intake of doses, from $76 \%$ risk reduction with 2 doses weekly, $96 \%$ with 4 doses weekly, to $99 \%$ with 7 doses per week (Anderson et al., 2012). In the PROUD study with 544 participants, follow-up for HIV incidence was completed for 243 of 259 patient-years in the immediate group and 222 of 245 in the deferred group. HIV incidence was 1.2/100 person-years in the immediate group and 9.0/100 person-years in the deferred group, yielding a relative reduction of $86 \%$. In one observational cohort study that focused on efficacy, safety and effect of sexual behaviour of on-demand PrEP for HIV in MSM that started between 2014-2015, 361 participants were recruited, after whom a follow-up was performed for 18.4 months. HIV incidence in this PrEP group was 0.19 per 100 person-years, which was much lower than that in the placebo group and suggested a PrEP efficacy of 97\% (Molina et al., 2017).

However, efficacy results on studies done with women have been inconsistent (Janes et al., 2018). In one study where concentrations of PrEP metabolites in pregnant women were investigated, results showed a 45\%-58\% decrease of plasma tenofovir (TFV) and tenofovir diphosphate (TFV-DP) in dried blood spots (DBS) from women who were in their second and third trimester of pregnancy compared to those from non-pregnant women (Pyra et al., 2018). In another study, reduced concentrations of TDF metabolites were found in vaginal tissues compared to that in rectal tissues (Koss et al., 2018). The intricate milieu of the female genital tract may be susceptible to factors such as hormonal changes, microbiome, and inflammation (Nicol, Corbino, \& Cottrell, 2018; Farcasanu \& Kwon, 2018; Karim et al., 2018), which may also affect the efficacy. Besides these potential gender-related differences, adherence has been considered to play a critical role in PrEP efficacy.

\section{Challenges to PrEP Adherence}

(1) Lack of awareness and trust

For PrEP to be efficient, people apparently need to be aware of it. Currently, not many people have enough information about this prevention method (Mayer et al., 2018), including those who work within health care sectors except HIV specialists. In one study conducted among pharmacy students, understanding that a HIV test is required prior to prescribe PrEP is lacking, even though they were familiar with prescription guidelines. It is 
surprising that well-educated professionals may still lack enough information. It indicates that knowledge about PrEP has been hindered from educating people who are sexually active and at risk of HIV (Przybyla et al., 2019).

Certain ethnic minorities in the United States have some conspiracy mindset about HIV/AIDS. Some of them believe that AIDS was developed by the government as a dark plan to wipe out their people. Others believe that their ethnic group have been unfairly used as experimental subjects by pharmaceutical corporations (Olansky et al., 2019). These conspiracy theories may contribute to subpar adherence to PrEP among these groups.

(2) High cost

Currently, the annual expense for PrEP in the United States is over 10,000 US dollars per individual. This cost will become significantly greater when clinical and laboratory monitoring expenses are added (Mayer et al., 2018; Sullivan \& Siegler, 2018). Individuals without insurance and those with insurance but unable to afford monthly co-pays may encounter financial constraints (Mayer et al., 2018). Only people who have earn above average can access PrEP more easily. The selling price of Truvada would have to be cut half for cost-effectiveness to most people (McCormack et al., 2016; Zhang et al., 2019). Some participants in another study reported that they would take PrEP if it was provided for free (Golub et al., 2013).

(3) Lack of established routine

It takes practice and commitment to adhere to daily routines. For PrEP to be effective, it has to be administered every day. Several factors may interfere the routine, including being away from home, busy work schedule (Karim et al., 2018), change of life style, lack of scheduled time, emotional instability, difficulty in purchase of drug, and so on (Hunt et al., 2019; Amico et al., 2019).

(4) Pressure and risk related to PrEP practice

Compared to other sexually transmitted diseases (STD) such as chlamydia and gonorrhoea, HIV has received more attention from almost every society. High-risk group who should take PrEP may encounter issues like deficiency of medicinal confidentiality, inconvenient transportation, inadequate clinic time and limited access to health care (Sharma et al., 2019). Furthermore, the phenomenon of MSM is still an unspeakable topic or illegal in certain regions. MSM who want to participate in PrEP may run into big trouble of moral judgement, even threatening of lives. Prostitution remains illegal in most countries, which hindered sex workers from effective preventative methods as well.

\section{Potential Solutions to Poor Adherence}

Poor adherence is the leading cause of low efficacy of PrEP, which absolutely needs creative solutions. Taking a dose every day for life long is indeed tedious. Production of long-release formulation and reduction of dose frequency is one of the approaches that can help alleviate this adherence challenge. Recently, new long-acting (LA) formulations have been invented. One implantable nanofluidic device with cabotegravir (CAB) is one of these new inventions (Figure 1), which was capable to reduce the dosing frequency from daily doses to bimonthly injections (Pons-Faudoa et al., 2019). CAB is currently under clinical trials.

(A)

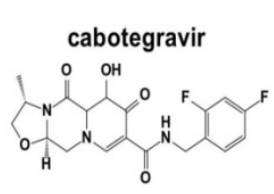

(B)

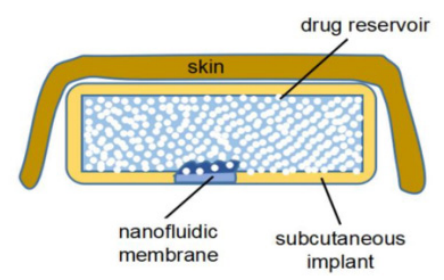

Figure 2. The chemical structure of $\mathrm{CAB}$ and its LA nanofluidic implant

Another subcutaneous LA formulation was also developed with tenofovir alafenamide (TAF) and FTC loaded nanoparticles (NPs) to solution in humanized (hu) mice. Efficacy of TAF+FTC NPs and TAF+FTC solution were proven to be dramatically different when hu-BLT mice were vaginally challenged with a transmission-founder virus. TAF+FTC NPs resulted in significant $(p=0.0002)$ protection from HIV-1, compared to the control. It 
indicated that detectable levels of TAF+FTC in vagina among TAF+FTC NP-treated mice were correlated with prolonged PrEP efficacy, which provides another new LA formulation for PrEP (Mandal et al., 2019).

Other methods to tackle this issue include adoption of smartphones and use of mobile apps (LeGrand et al., 2018). mSMART is one smartphone app. It not only sends daily reminder to PrEP users, but also connects people within a virtual society. Around 30\% improvement of sample adherence was seen as a result of mSMART use. In addition, users showed high satisfaction and willingness to recommend mSMART to others (Mitchell et al., 2018). Application of this type of apps is very promising and convenient in today's social media-cantered life. MyChoices is another theoretical prevention app designed especially for young MSM (Biello et al., 2019). The app will help users with testing reminders, location-based alerts, videos and infographics.

Recently, in the Project IMPACT study, researchers hypothesized another method to improve PrEP adherence by targeting pleasure loss in MSM. They suggested that counselling and advice to partake in safer sexual practices may help reduce HIV risk behaviorr and concurrent stimulant use (Mimiaga et al., 2018), therefore, increasing PrEP efficacy.

\section{Side Effects of PrEP}

The most general side effects associated with PrEP in uninfected individuals include headache, abdominal pain, weight loss, nausea, dizziness, vomit, and diarrhea (Tetteh et al., 2017; Desai et al., 2017). Some symptoms disappear after a while. Certain side effects may not need medical attention. Few severe side effects need to be taken care of or PrEP has to be ceased, including liver and kidney damages.

As listed in Table 2, FTC/TDF, FTC and FDF have been reported to cause different types of adverse effects, including in gastrointestinal, nervous, musculoskeletal, hematologic, dermatologic, genitourinary, metabolic, hepatic and renal systems. Besides, it may also trigger psychiatric disorder, such as anxiety and depression. For gastrointestinal tract, diarrhea, nausea (Desai et al., 2017), increased serum amylase, abdominal pain, and vomiting have been reported in large randomised controlled trials (Grant et al., 2010; Choopanya et al., 2013). Pancreatitis is possible but quite rare. For the nervous system, dizziness and headache (Desai et al., 2017) are quite common and somnolence is less common. For the musculoskeletal system, elevated creatine kinase was reported in up to $9 \%$ patients using Truvada. Decreased bone mineral density and bone fractures (Desai et al., 2017; Montjane, Dlamini, \& Dandara, 2018; Mulligan et al., 2015) have also been reported, especially for people with vitamin $\mathrm{D}_{3}$ deficiency, intake of Truvada would cause decrease of bone mineral density. Supplementation of vitamin D was suggested for PrEP users (Havens et al., 2019; Havens et al., 2019; Nanayakkara et al., 2019). In addition, back pain occurs with up to $10 \%$ frequency. iPrEx studies reported a significant decrease in bone mineral density in patients using FTC/TDF (Mulligan et al., 2015; Kasonde et al., 2014). For the hematologic system, decreased neutrophils (up to $13 \%$ ) is very common with FTC/TDF, and decreased hemoglobin occurs with up to $10 \%$ probability. Rash may happen in skins of Truvada users (Dando \& Wagstaff, 2004). For the genitourinary system, proteinuria, urethritis, urinary tract infection, haematuria, genital ulceration and anogenital warts were reported in patients using FTC/TDF. For metabolism, hyperglycaemia occurs with up to $10 \%$ possibility in patients using FTC/TDF. For liver, hepatic steatosis (Coutinho \& Prasad, 2013) and hepatitis were the two commonly reported side effects. For the renal system, increased creatinine level was observed for FTC/TDF users. In the real world, kidney impairment was found among TDF-treated HIV-positive patients, who sometimes experienced renal failure, Fanconi syndrome (Coutinho \& Prasad, 2013; Paxton, Hope, \& Jaffe, 2007), and proximal renal tubulopathy. At the same time, proteinuria accompanies creatinine elevation (Tang et al., 2018). In short, side effects of FTC/TDF have become a concern.

On the other hand, clinical studies assessing the effects of TDF/FTC usually had restricted inclusion criteria of participants, which potentially excluded people with other health issues and cannot represent the general population. Therefore, it is critical to evaluate the side effects among more heterogeneous populations, including those with comorbid conditions (such as hypertension, diabetes, asthma or depression, etc), and those under other medication (such as nonsteroidal anti-inflammatory drugs, birth-control drugs, etc). At the same time, PrEP users should take regular physical examination, in order to monitor renal and hepatic health, if not more frequent than others. 
Table 2. Summary of side-effect symptoms of PrEP

\begin{tabular}{|c|c|c|c|}
\hline Organ/System & FTC/TDF & FTC & TDF \\
\hline Gastrointestinal & $\begin{array}{l}\text { diarrhea, nausea, increased serum } \\
\text { amylase, abdominal pain, } \\
\text { vomiting, flatulence }\end{array}$ & none reported & diarrhea, vomiting, nausea, pancreatitis \\
\hline Neurological & dizziness, headache, somnolence & none reported & neuropathy, peripheral neuritis \\
\hline Musculoskeletal & $\begin{array}{l}\text { increased creatine kinase, } \\
\text { decreased bone mineral density, } \\
\text { back pain }\end{array}$ & none reported & rhabdomyolysis, muscular weakness, myopathy. \\
\hline Hematologic & $\begin{array}{l}\text { decreased haemoglobin, decreased } \\
\text { neutrophils }\end{array}$ & anaemia, neutropenia & decreased neutrophils \\
\hline Dermatologic & rash & rash & rash \\
\hline Genitourinary & $\begin{array}{l}\text { proteinuria, urethritis, urinary tract } \\
\text { infection, haematuria, genital } \\
\text { ulceration, anogenital warts }\end{array}$ & none reported & glycosuria, haematuria \\
\hline Metabolic & hyperglycaemia & $\begin{array}{l}\text { hyperglycaemia, } \\
\text { increased/decreased serum } \\
\text { glucose }\end{array}$ & hypophosphatemia \\
\hline Hepatic & hepatic steatosis, hepatitis & none reported & none reported \\
\hline Renal & $\begin{array}{l}\text { increased creatinine, renal failure, } \\
\text { Fanconi syndrome, proximal renal } \\
\text { tubulopathy }\end{array}$ & none reported & $\begin{array}{l}\text { renal failure, acute renal failure, Fanconi syndrome, } \\
\text { proximal renal tubulopathy, increased creatinine, } \\
\text { nephrogenic diabetes insipidus, acute tubular } \\
\text { necrosis }\end{array}$ \\
\hline
\end{tabular}

\section{Conclusion}

Ever since the approval of PrEP, many trials have been established to test its efficacy and safety. Most of these studies have been quite positive. PrEP efficacy results ranged between 44\%-97\%. However, its efficacy is dependent on adherence, and the latter is dependent on multiple factors of the society and each individual. For example, starting PrEP is not effective for people with undiagnosed early HIV infection. In addition, purchase of PrEP medicine online may skip the step of HIV testing, which is actually needed before use. Current administration methods, including daily oral dose, do not address these issues. Moral pressure, culture, inaccessible facilities, stagnant screening methods, poor monitoring strategies, unawareness of the drug and inadequate education to the people still hinder PrEP from reaching its ultimate efficacy.

More research on the development of advanced technology and attention to new methods that are promising to answer the adherence questions are needed, like the development of long-acting drugs, modernization of monitoring strategies and advanced screening approaches. One recent simulating use of Dolutegravir (DTC), a second-generation integrase inhibitor, indicated that prophylaxis with 50mg DTG was non-inferior to Truvada and that it may outperform the latter if good medical adherence is in daily practice (Duwal, Dickinson, Khoo, \& von Kleist, 2018). HIV vaccine remains as the hot spot for researchers, in spite of no luck during vaccine development due to the incredible diversity of HIV strains (Mega, 2019). Another preventative method under investigation was use of vaginal ring among high-risk women, by releasing antiretroviral drugs into the vagina from a silicon band (Cohen, 2016). So far, the ring only provided modest protection for women between 21 and 45 years old, barely showing protection for younger or older females.

While working on these resolutions, some attention will needed to PrEP for pregnant women and cisgender women in general., The current drugs have shown some significant and non-negligible negative outcomes in this particular group, comparing to men and transgender women. Furthermore, no matter how effective the drug may be, there are adverse effects. PrEP is associated with certain adverse effects. Some are minor and some are severe, with medical intervention or complete discontinuation of the drug needed. As much as we are excited about PrEP, we cannot be oblivious to the definite and probable shortcomings brought by this drug.

\section{Conflict of interests}

The authors declare that there is no conflict of interests regarding the publication of this paper. 


\section{References}

Amico et al. (2019). Perspectives of US women participating in a candidate PrEP study: Adherence, acceptability and future use intentions. Journal of the International AIDS Society, 22(3), e25247.

Anderson et al. (2012). Emtricitabine-tenofovir concentrations and pre-exposure prophylaxis efficacy in men who have sex with men. Science Translational Medicine, 4(151), 151ra125-151ra125.

Baeten et al. (2012). Antiretroviral prophylaxis for HIV prevention in heterosexual men and women. New England Journal of Medicine, 367(5), 399-410.

Biello et al. (2019). A Mobile-Based App (MyChoices) to Increase Uptake of HIV Testing and Pre-Exposure Prophylaxis by Young Men Who Have Sex with Men: Protocol for a Pilot Randomized Controlled Trial. JMIR Research Protocols, 8(1), e10694.

Calabrese, S. K., Krakower, D. S., \& Mayer, K. H. (2017). Integrating HIV preexposure prophylaxis (PrEP) into routine preventive health care to avoid exacerbating disparities. American Journal of Public Health, 107(12), 1883-1889.

Choopanya et al. (2013). Antiretroviral prophylaxis for HIV infection in injecting drug users in Bangkok, Thailand (the Bangkok Tenofovir Study): A randomised, double-blind, placebo-controlled phase 3 trial. The Lancet, 381(9883), 2083-2090.

Cohen, J. (2016). Drug-laced vaginal ring succeeds against HIV-sometimes. Science.

Coutinho, B., \& Prasad, R. (2013). Emtricitabine/tenofovir (Truvada) for HIV prophylaxis. Am Fam Physician, 88(8), 535-540.

Dando, T. M., \& Wagstaff, A. J. (2004). Emtricitabine/tenofovir disoproxil fumarate. Drugs, 64(18), 2075-2082.

Desai et al. (2017). State of the art review: Recent advances in PrEP for HIV. BMJ (Clinical research ed.), 359, j5011.

Duwal, S., Dickinson, L., Khoo, S., \& von Kleist, M. (2018). Hybrid stochastic framework predicts efficacy of prophylaxis against HIV: An example with different dolutegravir prophylaxis schemes. PLoS Computational Biology, 14(6), e1006155.

Farcasanu, M., \& Kwon, D. S. (2018). The Influence of Cervicovaginal Microbiota on Mucosal Immunity and Prophylaxis in the Battle against HIV. Current HIV/AIDS Reports, 15(1), 30-38.

Golub et al. (2013). From efficacy to effectiveness: Facilitators and barriers to PrEP acceptability and motivations for adherence among MSM and transgender women in New York City. AIDS Patient Care and STDs, 27(4), 248-254.

Grant et al. (2010). Preexposure chemoprophylaxis for HIV prevention in men who have sex with men. New England Journal of Medicine, 363(27), 2587-2599.

Havens et al. (2019a). Association of Vitamin D Insufficiency and Protective Tenofovir Diphosphate Concentrations with Bone Toxicity in Adolescent Boys and Young Men Using Tenofovir Disoproxil Fumarate/Emtricitabine for HIV Pre-Exposure Prophylaxis. AIDS Research and Human Retroviruses, 35(2), 123-128.

Havens et al. (2019b). Changes in Bone Mass After Discontinuation of Pre-Exposure Prophylaxis (PrEP) with Tenofovir Disoproxil Fumarate/Emtricitabine in Young Men Who Have Sex With Men: Extension Phase Results of Adolescent Trials Network Protocols 110 and 113. Clinical Infectious Diseases.

Hosek et al. (2013). Project PrEPare (ATN082): The acceptability and feasibility of an HIV pre-exposure prophylaxis (PrEP) trial with young men who have sex with men (YMSM). Journal of Acquired Immune Deficiency Syndromes (1999), 62(4).

Hunt et al. (2019). Challenges to PrEP use and perceptions of urine tenofovir adherence monitoring reported by individuals on PrEP. AIDS Care, 1-4.

Janes et al. (2018). Weighing the evidence of efficacy of oral PrEP for HIV prevention in women in Southern Africa. AIDS Research and Human Retroviruses, 34(8), 645-656.

Karim et al. (2018). The microbiome and HIV prevention strategies in women. Current Opinion in HIV and AIDS, 13(1), 81-87. 
Kasonde et al. (2014). Bone mineral density changes among HIV-uninfected young adults in a randomised trial of pre-exposure prophylaxis with tenofovir-emtricitabine or placebo in Botswana. PloS one, 9(3), e90111.

Koss et al. (2018). Similar tenofovir hair concentrations in men and women after directly observed dosing of tenofovir disoproxil fumarate/emtricitabine: Implications for preexposure prophylaxis adherence monitoring. Aids, 32(15), 2189-2194.

Krakower, D. S., \& Mayer, K. H. (2015). Pre-exposure prophylaxis to prevent HIV infection: Current status, future opportunities and challenges. Drugs, 75(3), 243-251.

LeGrand et al. (2018). Testing the Efficacy of a Social Networking Gamification App to Improve Pre-Exposure Prophylaxis Adherence (P3: Prepared, Protected, emPowered): Protocol for a Randomized Controlled Trial. JMIR Research Protocols, 7(12), e10448.

Mandal et al. (2019). Nanoencapsulation introduces long-acting phenomenon to tenofovir alafenamide and emtricitabine drug combination: A comparative pre-exposure prophylaxis efficacy study against HIV-1 vaginal transmission. Journal of Controlled Release, 294, 216-225.

Mayer et al. (2018). Evolving models and ongoing challenges for HIV pre-exposure prophylaxis implementation in the United States. Journal of Acquired Immune Deficiency Syndromes (1999), 77(2), 119.

McCormack et al. (2016). Pre-exposure prophylaxis to prevent the acquisition of HIV-1 infection (PROUD): Effectiveness results from the pilot phase of a pragmatic open-label randomised trial. The Lancet, 387(10013), 53-60.

Mega, E. R. (2019). 'Mosaic' HIV vaccine to be tested in thousands of people across the world. Nature, 572(7768), 165-166.

Mimiaga et al. (2018). A randomized controlled efficacy trial of behavioral activation for concurrent stimulant use and sexual risk for HIV acquisition among MSM: Project IMPACT study protocol. BMC Public Health, $18(1), 914$

Mitchell et al. (2018). Smartphone-Based Contingency Management Intervention to Improve Pre-Exposure Prophylaxis Adherence: Pilot Trial. JMIR mHealth and uHealth, 6(9), e10456.

Molina et al. (2015). On-demand preexposure prophylaxis in men at high risk for HIV-1 infection. New England Journal of Medicine, 373(23), 2237-2246.

Molina et al. (2017). Efficacy, safety, and effect on sexual behaviour of on-demand pre-exposure prophylaxis for HIV in men who have sex with men: An observational cohort study. The Lancet HIV, 4(9), e402-e410.

Montjane, K., Dlamini, S., \& Dandara, C. (2018). Truvada (emtricitabine/tenofovir) pre-exposure prophylaxis roll-out among South African university students: Lots of positives, but let us keep an eye on possible surprises. SAMJ: South African Medical Journal, 108(2), 79-81.

Mulligan et al. (2015). Effects of emtricitabine/tenofovir on bone mineral density in HIV-negative persons in a randomized, double-blind, placebo-controlled trial. Clinical Infectious Diseases, 61(4), 572-580.

Murnane et al. (2013). Efficacy of pre-exposure prophylaxis for HIV-1 prevention among high risk heterosexuals: Subgroup analyses from the Partners PrEP Study. AIDS (London, England), 27(13).

Nanayakkara et al. (2019). Effect of Vitamin D Supplementation on Bone Turnover Markers during HIV Pre-exposure Prophylaxis using Tenofovir Disoproxil Fumarate-Emtricitabine in Men who have Sex with Men. AIDS Research and Human Retroviruses.

Nicol, M. R., Corbino, J. A., \& Cottrell, M. L. (2018). Pharmacology of Antiretrovirals in the Female Genital Tract for HIV Prevention. The Journal of Clinical Pharmacology, 58(11), 1381-1395.

Olansky et al. (2019). PrEP Awareness in the Context of HIV/AIDS Conspiracy Beliefs Among Black/African American and Hispanic/Latino MSM in Three Urban US Cities. Journal of Homosexuality, 1-11.

Paxton, L. A., Hope, T., \& Jaffe, H. W. (2007). Pre-exposure prophylaxis for HIV infection: What if it works? The lancet, 370(9581), 89-93.

Pons-Faudoa et al. (2019). 2-Hydroxypropyl- $\beta$-cyclodextrin-enhanced pharmacokinetics of cabotegravir from a nanofluidic implant for HIV pre-exposure prophylaxis. Journal of Controlled Release.

Przybyla et al. (2019). Awareness, knowledge, and attitudes towards human immunodeficiency virus (HIV) pre-exposure prophylaxis (PrEP) among pharmacy students. Currents in Pharmacy Teaching and Learning. 
Pyra et al. (2018). Tenofovir and tenofovir-diphosphate concentrations during pregnancy among HIV-uninfected women using oral preexposure prophylaxis. Aids, 32(13), 1891-1898.

Sanchez, M. C., Vivancos, M. G., \& Moreno, S. G. (2017). Pre-exposure prophylaxis (PrEP) against HIV: Efficacy, safety and uncertainties. Farmacia hospitalaria: Organo oficial de expresion cientifica de la Sociedad Espanola de Farmacia Hospitalaria, 41(5), 630-637.

Sharma et al. (2019). Acceptability and Feasibility of Self-Collecting Biological Specimens for HIV, Sexually Transmitted Infection, and Adherence Testing Among High-Risk Populations (Project Caboodle!): Protocol for an Exploratory Mixed-Methods Study. JMIR Research Protocols, 8(5), e13647.

Siegler et al. (2018). The prevalence of pre-exposure prophylaxis use and the pre-exposure prophylaxis-to-need ratio in the fourth quarter of 2017, United States. Annals of Epidemiology, 28(12), 841-849.

Sullivan, P. S., \& Siegler, A. J. (2018). Getting pre-exposure prophylaxis (PrEP) to the people: Opportunities, challenges and emerging models of PrEP implementation. Sexual Health, 15(6), 522-527.

Tang et al. (2018). Changes in Kidney Function Associated With Daily Tenofovir Disoproxil Fumarate/Emtricitabine for HIV Preexposure Prophylaxis Use in the United States Demonstration Project. Journal of Acquired Immune Deficiency Syndromes (1999), 77(2), 193-198.

Tetteh et al. (2017). Pre-exposure prophylaxis for HIV prevention: Safety concerns. Drug Safety, 40(4), 273-283.

Zhang et al. (2019) Modelling the epidemiological impact and cost-effectiveness of PrEP for HIV transmission in MSM in China. AIDS and Behavior, 23(2), 523-533.

\section{Copyrights}

Copyright for this article is retained by the author(s), with first publication rights granted to the journal.

This is an open-access article distributed under the terms and conditions of the Creative Commons Attribution license (http://creativecommons.org/licenses/by/4.0/). 